East African Medical Journal Vol. 85 No. 9 September 2008

LOW SENSITIVITY OF T-CELLBASED DETECTION OF TUBERCULOSIS AMONGHIV CO-INFECTED TANZANIAN IN-PATIENTS

C. Seshadri, MD., Massachusetts General Hospital, Gray/Jackson 504, 55 Fruit Street, Boston, MA 02114, USA, L. O. Uiso, Kibong'oto National Tuberculosis Hospital, Sanya Juu, Tanzania, J. Ostermann, PhD., 125 Old Chem Building, Duke University, Durham, NC 27710, USA, H. Diefenthal, MD., H. J. Shao, MD., Kilimanjaro Christian Medical Centre, P. O Box 3010, Moshi, Tanzania, H. Y. Chu, MD., Beth Israel Deaconess Medical Centre, 330 Brookline Avenue, Boston, MA 02215, USA, D. M. Asmuth, MD., UC Davis Health System, 2315 Stockton Blvd., Sacramento, CA 95817, N. M. Thielman, MD., MPH., Duke University Medical Centre, DUMC Box 3152, Durham, NC 27710, USA, J. A. Bartlett, MD., Duke University Medical Centre, DUMC Box 3238, Durham, NC 27710, USA and J. A. Crump, MBChB., DTM\&H, Duke University Medical Centre, DUMC Box 3867, Durham, NC 27710, USA

Request for reprints to: Dr. C. Seshadri, Massachusetts General Hospital, Gray/Jackson 504, 55 Fruit Street, Boston, MA 02114, USA

\title{
LOW SENSITIVITY OF T-CELL BASED DETECTION OF TUBERCULOSIS AMONG HIV CO-INFECTED TANZANIAN IN-PATIENTS
}

\author{
C. SESHADRI, L. O. UISO, J. OSTERMANN, H. DIEFENTHAL, H. J. SHAO, H. Y. CHU, D. M. ASMUTH, \\ N. M. THIELMAN, J. A. BARTLETT and J. A. CRUMP
}

\begin{abstract}
Objective: To evaluate the performance of QuantiFERON-TB GOLD (QFTG) in a resource-poor setting among patients with and without HIV infection.

Design: Cross-sectional study.

Setting: Two hospitals in Northern Tanzania.

Subjects: Eighty three adult male and female inpatients.

Intervention: All patients were screened for HIV infection and underwent tuberculin skin test (TST) and QFTG.

Results: Eighty-three subjects were enrolled, and 29 (35\%) of 83 were HIV-infected. QFTG yielded indeterminate results in $12(22 \%$; $95 \%$ CI $12 \%-34 \%)$ of $54 \mathrm{HIV}$-uninfected and $13(45 \%$; $95 \%$ CI $26 \%-64 \%)$ of 29 HIV-infected subjects ( $p=0.0323)$. Among those with smear-positive pulmonary tuberculosis, TST was positive in $40(100 \%$; $95 \%$ CI 91\%-100\%) of 40 HIV-uninfected subjects compared with seven $(54 \%$; $95 \%$ CI $25 \%$ $81 \%$ ) of $13 \mathrm{HIV}$-infected subjects ( $\mathrm{p}<0.0001$ ), and QFTG was positive in $28(70 \%$; $95 \% \mathrm{CI}$ $53 \%-83 \%)$ of $40 \mathrm{HIV}$-uninfected subjects compared with three (23\%; 95\% CI 5\%-54\%) of $13 \mathrm{HIV}$-infected subjects $(\mathrm{p}=\mathbf{0 . 0 0 2 9})$. Among medical inpatients at risk for latent tuberculosis infection, TST was positive in seven $(50 \%)$ of $14 \mathrm{HIV}$-uninfected patients and three $(19 \%)$ of 16 HIV-infected patients $(\mathrm{p}=0.0701)$ and QFTG was positive among two $(14 \%)$ of $14 \mathrm{HIV}$-uninfected patients and three (19\%) of $16 \mathrm{HIV}$-infected patients $(\mathrm{p}=0.7437)$.

Conclusions: The presence of HIV co-infection was associated with a significant reduction in sensitivity of both the TST $(p<0.0001)$ and QFTG $(p=0.0029)$ for the diagnosis of active M.tuberculosis infection. The high proportion of indeterminate QFTG and lack of sensitivity, particularly among HIV-infected patients, may limit its applicability in settings like Tanzania. Larger studies in resource-poor settings are required.
\end{abstract}

\section{INTRODUCTION}

Tuberculosis is a leading killer of HIV-infected Africans and accounts for about a third of AIDS deaths worldwide. HIV-infected patients will develop active $\mathrm{TB}$ at a higher rate than those who are not infected with HIV(1) and nosocomial transmission of drug-resistant tuberculosis has been shown to be a major source of mortality among HIV-infected patients in South Africa (2). Therefore, diagnosis and treatment of latent tuberculosis infection (LTBI) is critical among hospitalised patients in endemic areas. Tuberculin skin testing (TST), the current standard of care for diagnosing latent tuberculosis 
infection, reflects technology from the early part of the $20^{\text {th }}$ century and is plagued by several limitations including poor specificity, limited sensitivity, the need for a return visit to perform skin test reading, and variability inherent in its application and reading (3). Furthermore, these limitations are magnified in the immunosuppressed host. Malnutrition alone causes false negative results and HIV-infected subjects have significantly depressed delayed typehypersensitivity responses resulting in up to $50 \%$ of co-infected persons testing "negative" with TST, whether $5 \mathrm{~mm}$ or $10 \mathrm{~mm}$ threshold is used (4).

Recently, assays that measure IFN- $\gamma$ cytokine production in response to in vitro stimulation of lymphocytes with $M$. tuberculosis derived antigen $(5,6)$ have been developed. QuantiFERON-TB GOLD (QFTG; Cellestis Inc, Carnegie, Australia) is a commercially available whole blood assay that measures IFN- $\gamma$ response to stimulation with ESAT- 6 and CFP-10, peptides specific for $M$. tuberculosis. It was approved by the U.S. Food and Drug Administration in 2004 for the diagnosis of latent tuberculosisinfection and has demonstrated increased specificity in bacille Calmette-Guérin (BCG) vaccine recipients compared to TST. Because of the lack of a gold standard for the diagnosis of LTBI, sensitivity of QFTG has been assessed based on detection of patients with active tuberculosis (7). However, limited data exist regarding its use in HIV-infected patients $(8,9)$ and in resourcepoor settings $(10,11)$. Furthermore, there are no published studies on the use of QFTG in hospitalised patients in countries where TB is endemic.

Since HIV co-infection represents the greatest risk factor for development of active tuberculosis and reduces the sensitivity of diagnostic methods used routinely in resource-poor settings, we sought to evaluate the utility of QFTG in Tanzania, a country with high prevalence of both $M$. tuberculosis and HIV infection as well as a national standard of BCG vaccination. Our study was designed to answer two questions. First, what are the performance characteristics among general inpatients at risk for latent tuberculosis infection (LTBI) in a country where theincidence of TB is high $(347 / 100,000$ person/ year) and HIV co-infection (36\%) is common (12). Second, what is the sensitivity of QFTG among HIV-infected hospitalised patients using smear positive pulmonary TB as a diagnostic gold standard?.

\section{MATERIALS AND METHODS}

Study participants: All subjects were recruited from two hospitals in northern Tanzania: Kilimanjaro Christian Medical Centre (KCMC) and Kibong'oto National Tuberculosis Hospital (KNTH) between March and May, 2004. Subjects at KCMC were screened for entry based on age $>18$ years, expected inpatient status $>48$ hours, and referral by their primary team for inpatient HIV voluntary counseling and testing (VCT). Subjects at KNTH were screened for entry based on age $>18$ years, first diagnosis of smear positive pulmonary $\mathrm{TB}$, and treatment duration less than 42 days. Written informed consent was obtained from all subjects or their next of kin. KCMC subjects were asked about risk factors for exposure to TB including known household contact (defined as living with someone with active tuberculosis), presence and duration of cough, and previous or current treatment for TB. Chest radiograph (CXR) results within three months of presentation were solicited from the medical record, if not obtained on admission. CXR were reviewed by a single attending radiologist and assessed for the number of abnormal segments (0-6). All subjects were assessed for the presence of BCG vaccination scar over the deltoid. Information on semi-quantitative AFB smear results of KNTH subjects was obtained from their current medical record. Patients were subgrouped into TB Patients (those with documented smear positive pulmonary tuberculosis) and LTBI/BCG Patients (medical inpatients at risk for latent tuberculosis infection and history of BCG vaccination).

Sample collection and TST: A heparinised blood sample (5-10 ml) was collected by venipuncture, stored at room temperature, and processed for QFTG within twelve hours. Blood was collected prior to placement of tuberculin skin test (TST) by the Mantoux method. Briefly, 0.1ml (5TU) Tubersol reagent (Connaught Laboratories Inc, Toronto, Ontario) was injected intradermally into the volar aspect of the forearm and transverse / longitudinal induration diameter was measured 48-72 hours later by a principal investigator. For all subjects, a positive TST was defined as $\geq 10 \mathrm{~mm}$ unless the subject was HIV-infected, then the cutoff was $\geq 5 \mathrm{~mm}$.

Whole blood IFN- $\gamma$ assay: The whole blood IFN- $\gamma$ assay, QuantiFERON-TB GOLD [QFTG] (Cellestis Inc., Carnegie, Australia) was used. The recently updated QFTG 'in-tube' was not available at the time. Assays were performed according to manufacturer's instructions except that IFN $-\gamma$ standards were measured in duplicate at 1:1 (8 IU/ml), 1:3, 1:9, 1:27, $1: 81$, and Nil concentrations on each plate as opposed to $1: 1(4 \mathrm{IU} / \mathrm{ml}), 1: 4,1: 16$, and Nil as recommended by the manufacturer. This was done because the correlation coefficient of the standard curve was not consistently robust, and we had a limited amount of IFN- $\gamma$ standard with which to troubleshoot in the field. Importantly, all predefined quality control measures were met for each patient sample. ELISA plates were read with Dynex MRX II and associated Dynex Revelation software (Chantilly, VA) was used to interpolate IFN- $\gamma$ concentrations from optical density measurements.

HIV testing: Prior to HIV testing, all subjects underwent appropriate pretest counseling provided 
by the local nursing staff. For subjects at KCMC, testing was performed using serum samples obtained after stimulation with Nil antigen and subsequent freezing at $-80^{\circ} \mathrm{C}$. Samples were brought to room temperature, vortexed, and then tested. For subjects at $\mathrm{KNTH}$, testing was performed on whole heparinised blood.

In both groups, rapid agglutination assays using World Health Organisation (WHO) recommended protocol were applied. All samples first underwent Determine HIV1/2 (Abbott Labs, Germany). If indeterminate or positive, confirmatory testing was done with Capillus HIV-1/HIV-2 (Trinity Biotech, Ireland). Subjects at KCMC also had their blood tested in the hospital clinical laboratory by ELISA (Biomerieux, Netherlands). All subjects received the results of their tests and underwent post-test counseling.

Statistical analysis: Data were entered using Microsoft Excel (Microsoft Inc., Redmond, WA) and analysed using Stata version 9.2 (Stata Corporation, College Station, TX). Differences in proportions were analysed using two-sample proportions tests for binomial probabilities; differences in the distribution of continuous variables were analysed using Wilcoxon's rank sum test. Odds ratios for differences in proportions and sensitivities, and their
95\% confidence intervals, were calculated using the Koopman method (13). Significance was evaluated at $\mathrm{p}=0.05$ using two-sided tests. Based on $87 \%$ sensitivity of QFTG in healthy patients according to the manufacturer, we estimated a sample size of 100 would allow us to detect a clinically significant change of at least $7-10 \%$.

Ethics: The research protocol was approved by an Institutional Review Board of Duke University Medical Centre, the Kilimanjaro Christian Medical Centre Research Ethics Committee, and the Tanzania National Institute of Medical Research Coordinating Committee.

\section{RESULTS}

We approached 100 patients and enrolled 83 in our study (Table 1). Fourty seven (57\%) of 83 enrolled patients were male and the median age was 36 years (range $20-73$ years). Patients were subgrouped into TB Patients (those with documented smear positive pulmonary tuberculosis; $n=53$ ) and LTBI/ BCG Patients (medical inpatients at risk for latent tuberculosis infection and history of $B C G$ vaccination; $\mathrm{n}=30)$. Proportions of TB patients $(\mathrm{n}=53)$ and LTBI/ BCG Patients ( $\mathrm{n}=30$ ) with HIV infection (Figure 1).

Table 1

Clinical characteristics of study subjects stratified by tuberculosis diagnosis

\begin{tabular}{|c|c|c|c|}
\hline & $\begin{array}{l}\text { TB Patients }(\mathrm{n}=53) \\
\text { No. }(\%)\end{array}$ & $\begin{array}{c}\text { LTBI/BCG Patients }(\mathrm{n}=30) \\
\text { No. } \quad(\%)\end{array}$ & P-value \\
\hline Age in years: mean (sd) & 16.34 & 8.45 & $0.8052 \mathrm{~b}$ \\
\hline Female no. $(\%)$ & 30.2 & 66.7 & $0.0013 a$ \\
\hline HIV-infected no. (\%) & 24.5 & 53.3 & $0.0082 a$ \\
\hline BCG scar present no. $(\%)$ & 77.4 & 93.1 & $0.1505 \mathrm{a}$ \\
\hline Duration of treatment in days (sd) & 24.214 .7 & $\mathrm{n} / \mathrm{a}$ & \\
\hline LTBI/BCG Patients & HIV-infected & HIV-negative & $P$-value \\
\hline No. & 16 & 14 & \\
\hline Household TB contact $(\%)$ & 21 & 19 & $0.8548 \mathrm{a}$ \\
\hline $\begin{array}{l}\text { Median duration of cough in days } \\
\text { (range 0-720) }\end{array}$ & 37 & 0 & $0.0314 \mathrm{~b}$ \\
\hline $\begin{array}{l}\text { Median abnormal segments on chest } \\
\text { radiography (range 0-6) }\end{array}$ & 2 & 0 & $0.1663 b$ \\
\hline
\end{tabular}

(a) Two-sample binomial proportions test.

(b) Wilcoxon rank-sums

* Four patients without chest radiographs were excluded from the calculations 
Figure 1

Patient enrollment and stratification

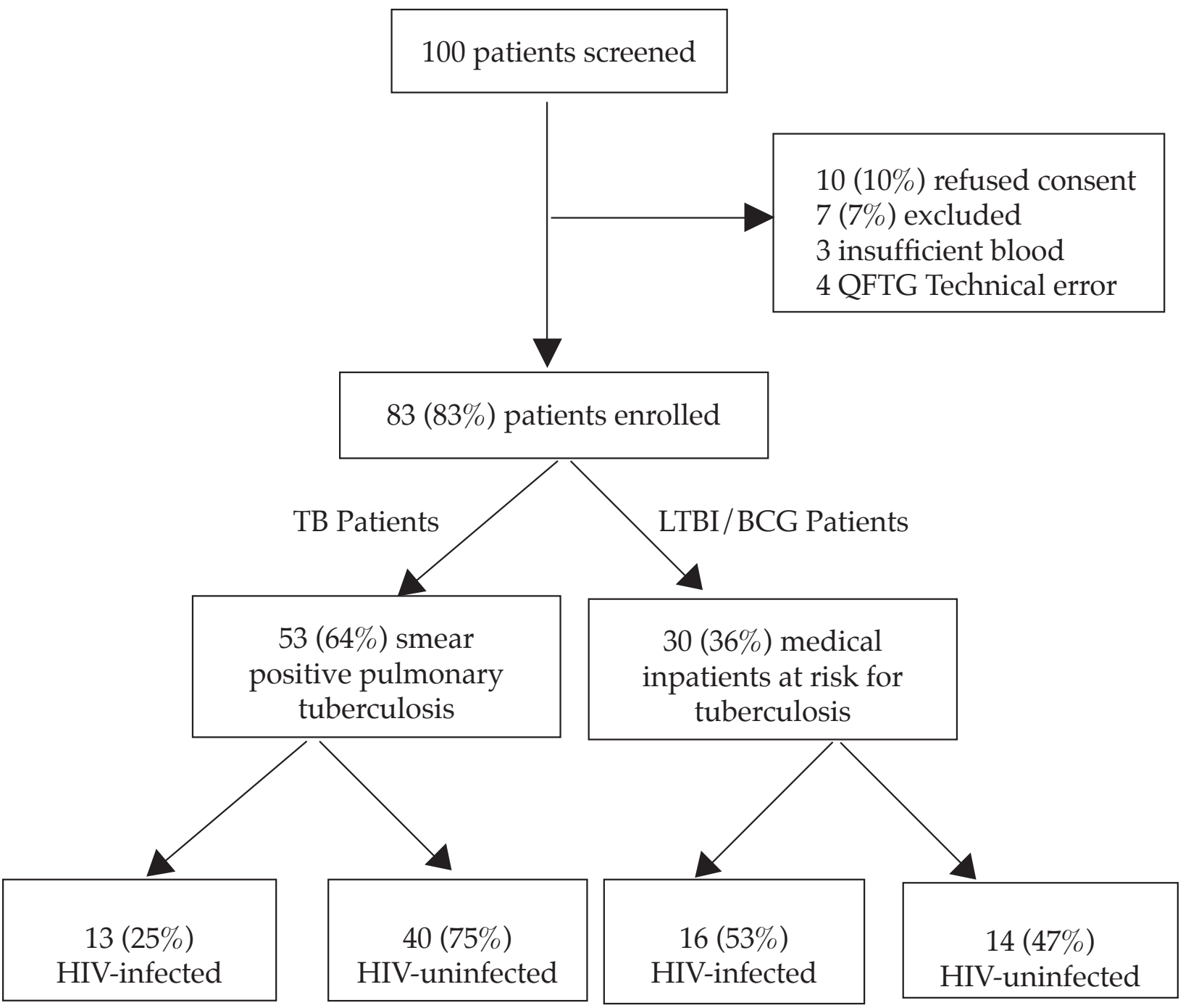

TB Patients had documented smear positive pulmonary tuberculosis as demonstrated by two $(2 / 53)$ or three $(51 / 53)$ semi-quantitative AFB smears. Subjects received a standard regimen of fourdrug antituberculous therapy (isoniazid, rifampin, ethambutol, and pyrazinamide) for a median of 26 days (IQR 11-39), and this duration of therapy did not differ by HIV serostatus $(p=0.9669)$. The remainder of enrolled patients (LTBI/BCG Patients) were more often female and had a higher incidence of HIV-infection than TB Patients. Analyses of epidemiologic, clinical, and radiographic findings from LTBI/BCG patients are listed in Table 1, stratified by HIV serostatus. Only the median duration of cough was significantly different; higher among HIV-infected patients. Most often, LTBI / BCG Patients were hospitalised for reasons unrelated to tuberculosis and did not routinely undergo sputum testing.

Among all patients, TST was positive among 47 (87\%;95\% CI 75\%-95\%) of 54 HIV-uninfected patients and $10(35 \% ; 95 \% \mathrm{CI} 18 \%-54 \%)$ of 29 HIV-infected patients $(\mathrm{OR}=2.52 ; 95 \% \mathrm{CI} 1.62-4.40 ; \mathrm{p}<0.0001)$. QFTG was positive among $30(56 \%$; 95CI $41 \%-69 \%)$ of 54 HIV-uninfected patients and $6(21 \%$; $95 \%$ CI $8 \%-40 \%)$ of 29 HIV-infected patients $(\mathrm{OR}=2.68 ; 95 \% \mathrm{CI} 1.37-5.82$; $\mathrm{p}=0.0022)$. QFTG yielded indeterminate results in $12(22 \% ; 95 \%$ CI $12 \%-34 \%)$ of 54 HIV-uninfected and $13(45 \% ; 95 \% \mathrm{CI} 26 \%-64 \%)$ of $29 \mathrm{HIV}$-infected subjects $(\mathrm{p}=0.0323)$ (Figure 2). 
Figure 2

Performance of QFTG and TST among all patients

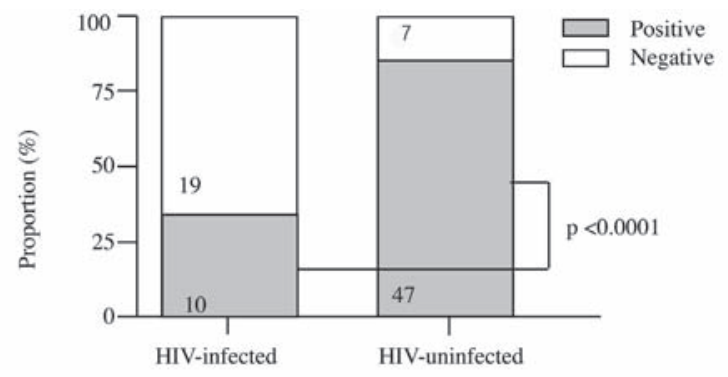

TST

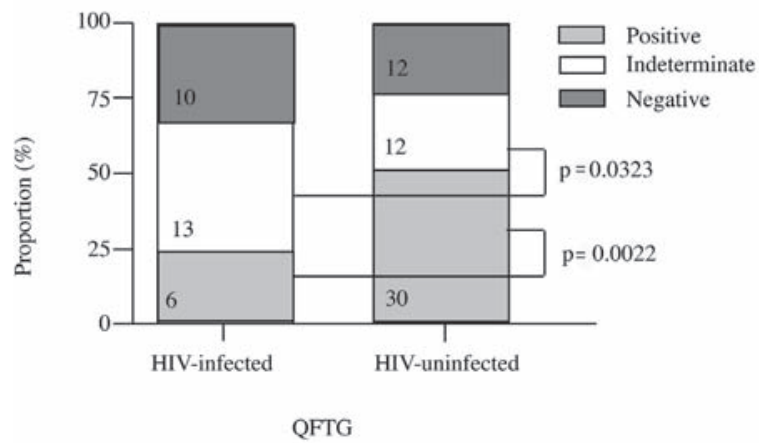

Among LTBI/BCGPatients, TST was positive among seven $(50 \%)$ of $14 \mathrm{HIV}$-uninfected patients and three $(19 \%)$ of 16 HIV-infected patients $(\mathrm{p}=0.0701)$. QFTG was positive among two (14\%) of $14 \mathrm{HIV}$-uninfected and three $(19 \%)$ of $16 \mathrm{HIV}$-infected patients $(\mathrm{p}=0.7437)$ (Figure3).Indeterminate QFTG responses werenoted in $12(40 \%)$ of 30 patients in this group.

Among TB Patients, TST was positive in 40(100\%; 95\% CI 91\%-100\%) of $40 \mathrm{HIV}$-uninfected subjects compared with seven $(54 \%$; 95\% CI 25\%-81\%) of 13 HIV-infected subjects (OR=1.86; 95\%CI 1.28- 3.39; $\mathrm{p}<0.0001)$, and QFTG was positive in $28(70 \%$; $95 \% \mathrm{CI}$ 53\%-83\%) of $40 \mathrm{HIV}$-uninfected subjects compared to three $(23 \%$; $95 \%$ CI $5 \%-54 \%)$ of 13 HIV-infected subjects $(\mathrm{OR}=3.03 ; 95 \% \mathrm{CI} 1.34-8.69 ; \mathrm{p}=0.0029)$ (Figure 4). QFTG was less sensitive than TST in detecting subjects with smear positive pulmonary tuberculosis among HIV-uninfected (OR=0.7; 95\% CI 0.55-0.84; $\mathrm{p}=0.0002)$ but not in HIV-infected (OR=0.43; 95\% CI $0.14-1.18 ; \mathrm{p}=0.1069)$ subjects. When restricting our analysis to patients with smear positive pulmonary tuberculosis and a treatment duration of 14 days or less, QFTG was positive among 13(72\%) of 18 patients, with $11(84 \%$; $95 \%$ CI $55 \%-98 \%)$ of 13 HIVuninfected patients and two $(40 \% ; 95 \% \mathrm{CI} 5 \%-85 \%)$ of five HIV-infected patients (OR=2.11;95\% CI0.98-7.30; $\mathrm{p}=0.0583$ ) having positive QFTG results. QFTG and TST showed modest agreement among all subjects $(67.47 \%)$ (Table 2).
Figure 3

Performance of QFTG and TST among medical in-patients at risk for tuberculosis

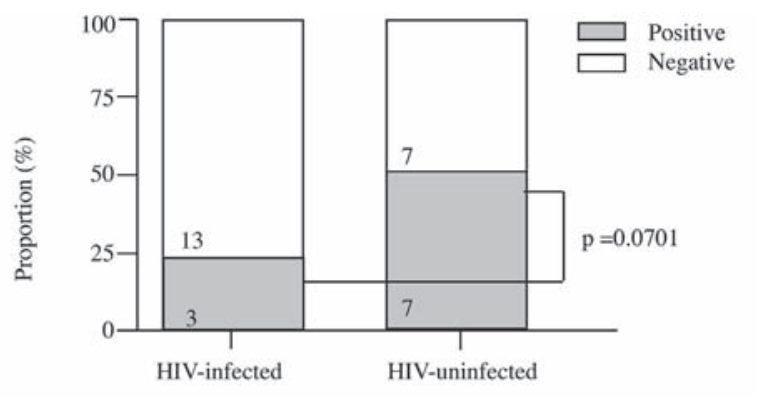

TST

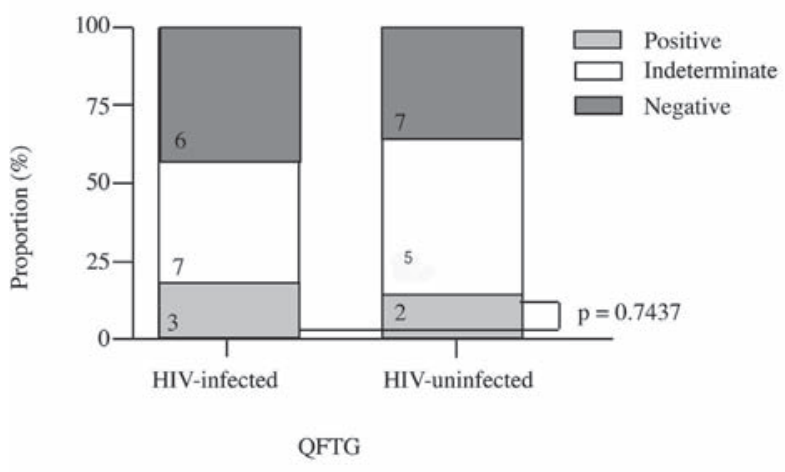

Figure 4

Performance of QFTG and TST in -patients with smear positive pulmonary tuberculosis
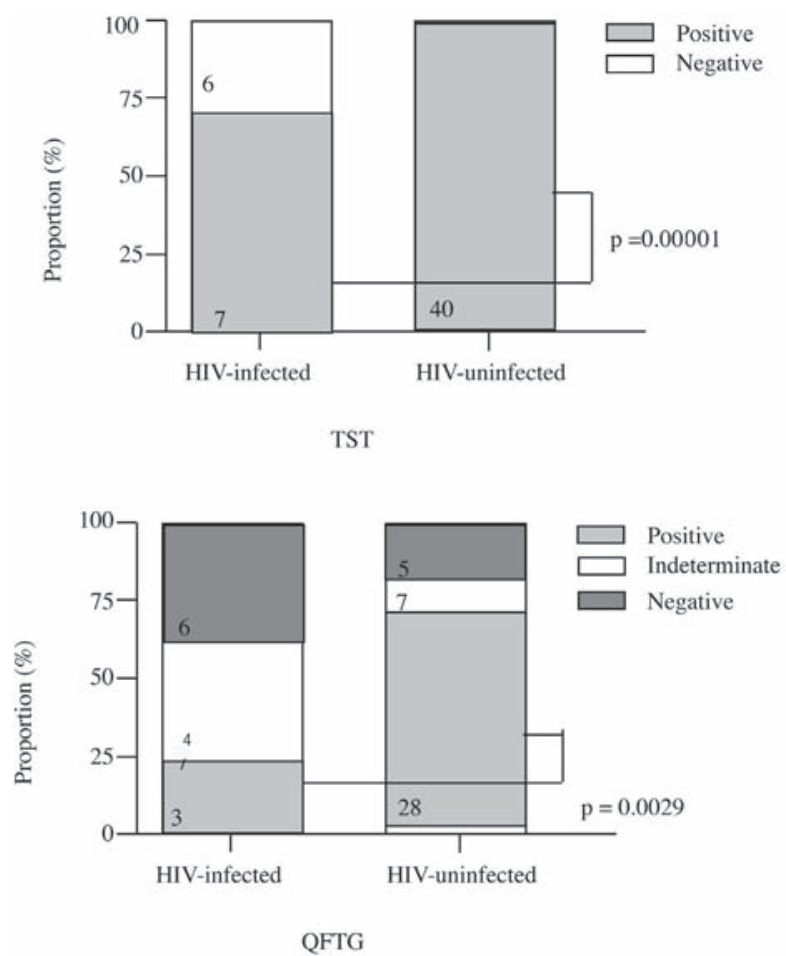
Table 2

Agreement between QFTG and TST among all HIVinfected and uninfected subjects

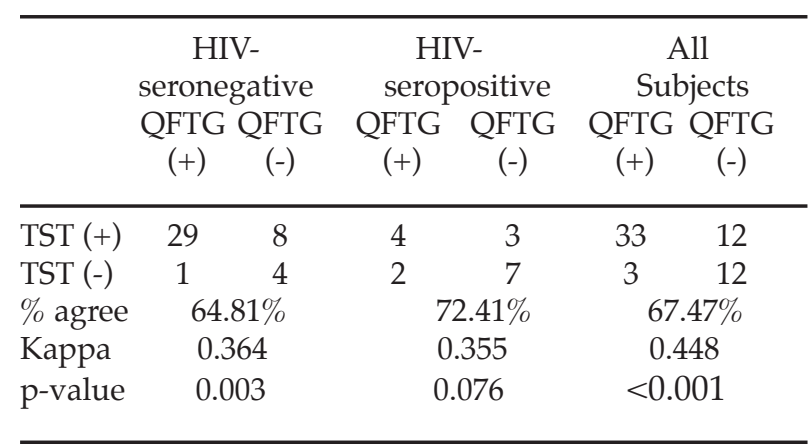

Most often, results were concordant (53\%) or QFTG result was indeterminate (30\%). QFTG was negative in eight smear positive subjects (six HIV-uninfected and two HIV-infected), and indeterminate in nine additional subjects (six HIV-uninfected and three HIV-infected). In only one subject did QFTG detect pulmonary TB when TST was falsely negative.

\section{DISCUSSION}

We demonstrate that QFTG is less sensitive than TST in detecting pulmonary tuberculosis in both HIVinfected and HIV-uninfected hospitalised patients in Tanzania. We also demonstrate a high proportion of indeterminate QFTG results among both HIVinfected $(45 \%)$ and HIV-uninfected $(22 \%)$ patients. In contrast to published studies, we present the results of implementing QFTG in a country with high prevalence of tuberculosis wherehospitalised patients were systematically screened for the presence of HIV co-infection. The large number of indeterminate QFTG even among HIV-uninfected hospitalised patients has immediate implications for the use of QFTG as a screening tool in the resource-limited nosocomial setting.

HIV co-infection markedly reduced thesensitivity of TST and QFTG to detect smear positive pulmonary tuberculosis by $46 \%(p<0.0001)$ and $67 \%(p=0.0029)$ respectively. Thelargenumber ofQFTG indeterminate responses were the result of sub-threshold responses to a positive control antigen (phytohemagglutinin), suggestive of global immunosuppression. A recently published study conducted in South Africa noted indeterminate QFTG results in five $(7 \%)$ of 74 HIV-infected and two (2\%) of 84 HIV-uninfected subjects (14). However, this study was conducted in ambulatory (not hospitalised) patients and the median CD4 count of HIV-infected participants was $392 / \mathrm{mm}^{3}$. Indeterminate responses were more likely if CD $4<100 / \mathrm{mm}^{3}$, which has been noted previously (8). Unpublished data from Kibong'oto National Tuberculosis Hospital in Tanzania reveal a median CD4 count of 104 cells / mm3 in HIV-infected patients with tuberculosis (15), suggesting our patients were more immunocompromised than those in published studies. In another study from South Africa, 23(15\%) of 154 patients with culture-confirmed pulmonary tuberculosishad indeterminateQFTG results, but they were not systematically screened for the presence of HIV co-infection (11). CD4 counts are not routinely available in resource-poor settings such as ours, though this information could help stratify those for whom QFTG would be appropriate.

According to the manufacturer, the overall sensitivity of QFTG is $87.6 \%$ in culture-confirmed pulmonary tuberculosis. In Tanzania, mycobacterial culture facilities are not routinely available, so we included patients with acid fast bacilli on sputum microscopy and a clinical picture consistent with tuberculosis, criteria which would merit treatment under the National Tuberculosis Programme. Only $70 \%$ of HIV-uninfected subjects with smear positive pulmonary tuberculosis had positive QFTG results in our study, and a number of factors could contribute to this reduced sensitivity. First, it is possible that some of the HIV-uninfected patients were infected with non-tuberculous mycobacteria (NTM), resulting in a falsely reduced sensitivity of QFTG. However, the predictive value of expectorated sputum microscopy for diagnosis of pulmonary tuberculosis is high even in settings where NTM are prevalent (16). Second, the median duration of antituberculous therapy in our cohort was 26 days, and prolonged treatment duration has been suggested to increase TST but reduce IFN$\gamma$ responses. In fact, we observed $100 \%$ sensitivity of TST compared to 70\% with QFTG ( $p=0.0002)$. Among patients with smear positive pulmonary tuberculosis who received treatment less than 14 days, we did not observe a difference in the rate of QFTG positivity ( $p=0.0583$ ) by HIV status; however, our sample size was not large enough to determine the effect of duration of tuberculosis therapy on QFTG performance. In a recent longitudinal study of the effect of LTBI treatment, it was noted that antigen specific T-cell responses increased 1.8 fold, peaking at 26 days before decreasing by the end of the treatment period (14). Additionally, health care workers in India treated for LTBI demonstrated positive QFTG results up to ten months after completing a course of treatment (17). Thus, while treatment duration may explain the discordance in sensitivity of QFTG relative to TST in HIV-uninfected patients, it is not clear that it would necessarily decrease the absolute sensitivity. Third, the nutritional status of the subjects, concomitant medical illness, and the presence of active TB itself, which has been shown to abrogate IFN- $\gamma$ cytokine responses (18), may have had an effect. Finally, we do not believe that deviation from manufacturer's recommendations in analysing IFN- $\gamma$ standards affected the sensitivity of the assay since all predefined quality control criteria were met. 
We noted a statistically significant difference in the proportion of HIV-infected patients among the two groups recruited from different hospitals which reflects the referral bias to a national tuberculosis hospital (KNTH) and local referral hospital (KCMC). Since KCMC is a referral hospital whose patients carry a very high level of acuity, the presence of malnutrition was a major confounding variable and additional clinical information such as body mass index or admitting diagnoses could have helped further stratify TB risk in this group. The characteristics of enrolled patients were not suitable to assessing specificity of QFTG, and this was not one of our stated objectives since high specificity has been previously demonstrated in healthy BCG-vaccinated subjects (7). However, we observed modest agreement (64.81\%; $\kappa=0.364 ; \mathrm{p}=0.003$ ) between TST and QFTG among HIV-uninfected patients despite routine BCG vaccination and possible infection with nontuberculous mycobacteria. A competing technology, the T-SPOT.TB (Oxford Immunotech, Oxford, UK) ELISPOT assay, appears to have a high sensitivity for identifying $M$. tuberculosis infection in HIV co-infected patients $(19,20)$, and direct comparison with QFTG suggests moderate agreement $(\kappa=0.37)$ between the tests(10).

Our experience demonstrates that QFTG can be successfully performed in resource-poor settings; however, the technical training required is multifaceted and includes proper venipuncture and tissue culture technique, performance and reading of ELISA plates, and complex quality control and data analysis. Routine use of QFTG among medical inpatients at risk for LTBI yielded a large number of indeterminate results, and the sensitivity of QFTG was markedly reduced by the presence of HIV co-infection. Despite the best of circumstances, the reduced sensitivity of QFTG observed in this report highlights the limitations of any immunologically based assay: reliance on an intact immunologic response in the setting of acute disease or concomitant illness in order to give positive results. That assumption is particularly problematic in the background of HIV infection, malnutrition, and other co-infections common in developing world contexts where TB is endemic. The results of this investigation should be confirmed by larger studies in hospitals in resourcepoor settings.

\section{ACKNOWLEDGEMENTS}

To Dr. John F. Shao for his administrative assistance, Dr. Chris Drakeley for technical assistance, and the nursing and administrative staff at KCMC and KNTH without whom this work would have beenimpossible. This work was presented in part at $3^{\text {nd }}$ International AIDS Society Conference on HIV Pathogenesis and
Treatment, Rio de Janiero, Brazil, July 24-27, 2005. Abstract TuPe7.1C09.

Grant Support: Drs. Bartlett and Thielman are supported by the AIDS Clinical Trials Group (U01AI39156), and Dr. Bartlett by a Mid-Career Investigator Award (K24-AI0174). Dr. Seshadri was supported by amfAR Travel Grant (\#106470-34-RGGN) for the duration of this study. Dr. Asmuth is supported by NIH R01AI051999-03.

Conflict of Interest Statement: All authors state no conflict of interest.

\section{REFERENCES}

1. Nunn, P., Williams, B., Floyd, K., et al. Tuberculosis control in the era of HIV. Nat. Rev. Immunol. 2005;5: 819-826.

2. Gandhi, N.R., Moll, A., Sturm, A.W., et al. Extensively drug-resistant tuberculosis as a cause of death in patients co-infected with tuberculosis and HIV in a rural area of South Africa. Lancet 2006; 368: 15751580 .

3. Lee, E. and Holzman, R.S. Evolution and current use of the tuberculin test. Clin. Infect. Dis. 2002; 34: 365-370.

4. Markowitz, N., Hansen, N.I., Wilcosky, T.C., et al. Tuberculin and anergy testing in HIV-seropositive and HIV-seronegative persons. Pulmonary Complications of HIV Infection Study Group. Ann. Intern. Med. 1993; 119: 185-193.

5. Lalvani, A., Pathan, A.A., McShane, H., et al. Rapid detection of Mycobacterium tuberculosis infection by enumeration of antigen-specific T cells. Am. J. Respir. Crit. Care Med. 2001;163: 824-828.

6. Streeton, J.A., Desem, N. And Jones, S.L. Sensitivity and specificity of a gamma interferon blood test for tuberculosis infection. Int. J. Tuberc. Lung Dis. 1998; 2: $443-450$

7. Mori, T., Sakatani, M., Yamagishi, F., et al. Specific detection of tuberculosis infection: an interferongamma-based assay using new antigens. Am. J. Respir. Crit. Care Med. 2004;170: 59-64.

8. Brock, I., Ruhwald, M., Lundgren, B., et al. Latent tuberculosis in HIV positive, diagnosed by the M. tuberculosis specific interferon-gamma test. Respir. Res. 2006; 7: 56.

9. Luetkemeyer, A.F., Charlebois, E.D., Flores, L.L., et al. Comparison of an interferon-gamma release assay with tuberculin skin testing in HIV-infected individuals. Am. J. Respir. Crit. Care Med. 2007;175: 737-742.

10. Rangaka, M.X., Wilkinson, K.A., Seldon, R., et al. Effect of HIV-1 infection on T-Cell-based and skin test detection of tuberculosis infection. Am. J. Respir. Crit. Care Med. 2007; 175: 514-520.

11. Tsiouris, S.J., Coetzee, D., Toro, P.L., et al. Sensitivity analysis and potential uses of a novel gamma interferon release assay for diagnosis of tuberculosis. J. Clin. Microbiol. 2006; 44: 2844-2850.

12. WHO. Country Profile: United Republic of Tanzania; 2006. 
13. Wang, D. sg 154: Confidence intervals for the ratio of two binomial proportions by Koopman's Method. Stata. Technical Bulletin. 2000;16-19.

14. Wilkinson, K.A., Kon, O.M., Newton, S.M., et al. Effect of treatment of latent tuberculosis infection on the T cell response to Mycobacterium tuberculosis antigens. J. Infect. Dis. 2006; 193: 354-359.

15. Shao, H.J., Crump, J.A., Ramadhani, H.O., et al. A randomized trial of early vs. delayed fixed dose combination Zidovudine/ Lamivudine/ Abacavir in patients co-infected with HIV and tuberculosis: Early findings of the tuberculosis and HIV immune reconstitution syndrome trial (THIRST). In: 13th conference on retroviruses and opportunistic infections; 2006; Denver, Colorado; 2006.

16. Yajko, D.M., Nassos, P.S., Sanders, C.A., et al. High predictive value of the acid-fast smear for Mycobacterium tuberculosis despite the high prevalence of Mycobacterium avium complex in respiratory specimens. Clin. Infect. Dis. 1994; 19: 334-336.

17. Pai, M., Joshi, R., Dogra, S., et al. Persistently elevated $\mathrm{T}$ cell interferon-gamma responses after treatment for latent tuberculosis infection among health care workers in India: a preliminary report. J. Occup. Med. Toxicol. 2006; 1: 7.

18. Zhang, M., Lin, Y., Iyer, D.V., et al. T-cell cytokine responses in human infection with Mycobacterium tuberculosis. Infect. Immun. 1995; 63: 3231-3234.

19. Liebeschuetz, S., Bamber, S., Ewer, K., et al. Diagnosis of tuberculosis in South African children with a Tcell-based assay: a prospective cohort study. Lancet. 2004; 364: 2196-2203.

20. Dheda, K., Lalvani, A., Miller, R.F., et al. Performance of a T-cell-based diagnostic test for tuberculosis infection in HIV-infected individuals is independent of CD4 cell count. Aids. 2005;19: 2038-2041. 